\title{
Implementation of clinical practice changes by experienced anesthesiologists after simulation- based ultrasound-guided regional anesthesia training
}

\author{
T. Edward Kim ${ }^{1,2}$, Toni Ganaway ${ }^{1,2}$, T. Kyle Harrison ${ }^{1,2}$, Steven K. Howard ${ }^{1,2}$, \\ Cynthia Shum $^{2}$, Alex Kuo ${ }^{1,2}$, and Edward R. Mariano, \\ ${ }^{I}$ Department of Anesthesiology, Perioperative and Pain Medicine, Stanford University School of Medicine, Stanford, \\ ${ }^{2}$ Anesthesiology and Perioperative Care Service, Veterans Affairs Palo Alto Health Care System, Palo Alto, CA, USA
}

\begin{abstract}
Background: Anesthesiologists who have finished formal training and want to learn ultrasound-guided regional anesthesia (UGRA) commonly attend 1 day workshops. However, it is unclear whether participation actually changes clinical practice. We assessed change implementation after completion of a 1 day simulation-based UGRA workshop.

Methods: Practicing anesthesiologists who participated in a 1 day UGRA course from January 2012 through May 2014 were surveyed. The course consisted of clinical observation of UGRA procedures, didactic lectures, ultrasound scanning, hands-on perineural catheter placement, and mannequin simulation. The primary outcome was the average number of UGRA blocks per month reported at follow-up versus baseline. Secondary outcomes included preference for ultrasound as the nerve localization technique, ratings of UGRA teaching methods, and obstacles to performing UGRA.

Results: Survey data from 46 course participants (60\% response rate) were included for analysis. Participants were (median [10th-90th percentile]) 50 (37-63) years old, had been in practice for 17 (5-30) years, and were surveyed 27 (10-34) months after their UGRA training. Participants reported performing 24 (4-90) blocks per month at follow-up compared to $10(2-24)$ blocks at baseline $(\mathrm{P}<0.001)$. Compared to baseline, more participants at follow-up preferred ultrasound for nerve localization. The major obstacle to implementing UGRA in clinical practice was time pressure.

Conclusions: Participation in a 1 day simulation-based UGRA course may increase UGRA procedural volume by practicing anesthesiologists.
\end{abstract}

Key Words: Change implementation, Nerve block, Perineural catheter, Regional anesthesia, Simulation, Ultrasound.

Corresponding author: Edward R. Mariano, M.D., M.A.S.

Anesthesiology and Perioperative Care Service, Veterans Affairs Palo

Alto Health Care System, 3801 Miranda Avenue (112A), Palo Alto, CA 94304, USA

Tel: 1-650-849-0254, Fax: 1-650-852-3423

Email: emariano@stanford.edu

ORCID: http://orcid.org/0000-0003-2735-248X

Received: August 30, 2016.

Revised: October 13, 2016 (1st); November 29, 2016 (2nd).

Accepted: November 29, 2016.

Korean J Anesthesiol 2017 June 70(3): 318-326

https://doi.org/10.4097/kjae.2017.70.3.318

\section{Introduction}

Performance of ultrasound-guided regional anesthesia (UGRA) can be described as a sequence of steps that requires integration of multiple technical and cognitive skills [1,2]. A successful UGRA procedure involves knowledge of anatomy, interpretation of sonoanatomy, needle manipulation, and visualization, and confirmation of local anesthetic or catheter tip adjacent to the target nerve(s). Other considerations include optimizing ergonomics, identifying anatomical variation, and

(c) This is an open-access article distributed under the terms of the Creative Commons Attribution Non-Commercial License (http://creativecommons.org/ licenses/by-nc/4.0/), which permits unrestricted non-commercial use, distribution, and reproduction in any medium, provided the original work is properly cited. 
recognizing complications such as intravascular or intraneural injection. A "learning curve" has been described for the acquisition of such regional anesthesia skills by residents [3-5]. For practicing anesthesiologists who have completed their formal training and want to learn UGRA, participation in $8 \mathrm{~h}$ of continuing medical education (CME) has been recommended [6]. Non-CME workshops and courses may offer similar education and are commonly hosted by sponsors, but similar to CME workshops, they vary widely in design. Curricular elements include lectures, ultrasound scanning on a live model, needling practice with manufactured or non-human tissue phantoms or human cadavers, simulation, or combinations of these factors [6-10]. However, to date, the optimal UGRA curriculum to train this population of learners, practicing anesthesiologists, and changes in his or her clinical practice after such training are unknown.

A strong case has been made to include simulation as part of the UGRA training for practicing anesthesiologists [11,12]. One recent prospective study showed that practicing anesthesiologists without prior UGRA experience can learn advanced ultrasound-guided perineural catheter insertion skills in 1 day if they complete a standardized simulation-based curriculum; however, the self-reported number of UGRA procedures that they perform in the year after the course did not change [12]. This negative assessment of clinical practice change in the study may have been biased by strict inclusion criteria (i.e., no formal regional anesthesia training and no UGRA in their routine anesthesiology practice) and the standardized non-adaptive format of the workshop. These results may not reflect the situation for the general population of practicing anesthesiologists who attend UGRA courses and workshops on their own, many of whom have had varying degrees of success in implementing regional anesthesia programs; who have likely at least attempted, if not incorporated, ultrasound [13]; and who tend to ask for specific instructions relevant to their practice. Thus, we designed this study of practicing anesthesiologists with varying backgrounds who participated in an actual non-CME, non-research simulation-based UGRA course to assess clinical practice change implementation in terms of UGRA procedural volume.

\section{Materials and Methods}

This study was approved by the local Veterans Affairs (VA) Research and Development Committee and received exemption from Institutional Review Board review. It was registered at http://www.clinicaltrials.gov (NCT02295423).

\section{Participants}

Past attendees of a non-CME, 1 day UGRA course conducted at a tertiary care, university-affiliated VA hospital simulation center from January 2012 through May 2014 were invited to participate in this study. Study participation was voluntary, with no remuneration. Those who completed both pre- and post-course surveys using the same instrument [12] were included; there was no other exclusion criterion. The participant population consisted of post-graduate anesthesiologists who were interested in learning UGRA and were able to take 1 day off work to attend a course at no cost to them. These courses were supported by corporate sponsors, Halyard Health (Alpharetta, GA, USA) and B Braun (Bethlehem, PA, USA), with unrestricted funding paid directly to the institution; attendees were invited to participate in the course by the sponsor. None of the workshop instructors or authors of this report received honoraria from the sponsors. Generally, workshop participants were experienced anesthesiologists, not novices, and had demonstrated to the sponsors that they were at least proficient in basic UGRA. The sponsors had no input with regard to course content or course educational materials; these materials were limited to the handbook, $A$ Visual Guide to Regional Anesthesia (Point of Care Essentials) [14], lecture handouts, and review articles $[15,16]$. Parts of the course were tailored to participants' skill level in UGRA (basic or advanced) based on their UGRA self-assessments, experience, and specific requests. The course was standardized to a maximum of three participants per day to allow individual attention and facilitate interactions with course faculty.

\section{Pre-course survey}

On the morning of the course, participants completed a printed, paper version of the pre-course survey before their training. The pre-course survey instrument has been previously described [12]. It included questions regarding participants' baseline characteristics: clinical practice (e.g., years of anesthesiology experience post-residency, average number of peripheral nerve blocks in a typical month, preference for nerve localization technique at specific block sites, and block complications), perceived obstacles to performing UGRA, and the effectiveness of the UGRA teaching methods. These pre-course surveys were collected on the same day, before the end of the UGRA course.

\section{Course description}

A full-day course $(8 \mathrm{~h})$ was offered three or four times per quarter. Each course began with a clinical observation session in a designated regional anesthesia procedure area ("block room"). Participants observed first case UGRA procedures in consenting 
patients, including perineural catheter insertion techniques, performed by the hospital's regional anesthesiology and acute pain medicine team. Next, a didactic session addressed anatomy, the basics of ultrasonography, and setting up a regional anesthesia program, including documentation and billing. The remainder of the course consisted of two workshops. Workshop 1, in the morning, involved real-time ultrasound scanning of a live human model. Each session was tailored to the individual anesthesiologist, based on experience and interests. For example, at the participants' request, the scanning workshop for one group could have included neuraxial blocks (e.g., paravertebral and lumbar plexus blocks) whereas another group would not have requested those procedures if they were not considered relevant to their clinical practice. Workshop 2, in the afternoon, consisted entirely of simulation-based training. The first part entailed iterative practice of needle visualization for single-injection and continuous UGRA using a porcine meat model [12] under the one-on-one direction of an expert proctor. The remainder consisted of UGRA crisis management scenarios (e.g., paresthesia, overly anxious patient, and local anesthetic systemic toxicity) using a high-fidelity mannequin simulator with a porcine meat model as a substitute for one of the lower extremities (a "hybrid" simulator) [11,12].

\section{Post-course survey}

For the post-course surveys, participants were recruited from a comprehensive list of past workshop attendees through electronic mail and/or telephone calls. A research assistant contacted and recruited all of the participants over a 2-month period (December 2014-January 2015). Participants were asked to complete a post-course online survey, including the same elements as the pre-course survey, via a secure online system (Qualtrics, Provo, UT, USA). Participants voluntarily provided informed consent at the beginning of the online survey and before they began to answer the post-course survey questions (Appendix). If participants did not complete the post-course online survey after the initial contact, the same research assistant emailed or called the participants with a request to complete the survey, either online or over the phone using the same survey tool administered by the research assistant.

\section{Outcomes}

The primary outcome was the average number of peripheral nerve blocks reported per month at follow-up, compared to baseline. Secondary outcomes were the reported preference for ultrasound guidance as the sole nerve localization technique, ratings of UGRA teaching methods, and obstacles to performing UGRA. Participants were also surveyed about their experiences with inadvertent vascular puncture and local anesthetic systemic toxicity (LAST).

\section{Sample size estimate}

There was no a priori sample size estimate because all of the past attendees of the UGRA course held at the facility from January 2012 through May 2014 were invited to participate in the study.

\section{Statistical analysis}

Statistical analyses were performed with NCSS-PASS (Kaysville, UT, USA) and R version 3.0.2 (R Foundation for Statistical Computing, Vienna, Austria; http://www.R-project.org/). The normality of the distribution was determined for all of the scale variables. Single comparisons of independent variables were performed using Student's $t$-test for normally distributed data; the Mann-Whitney $U$ test was used for continuous data with distributions that were other than normal. For the primary outcome, a within-group comparison of average number of blocks at follow-up versus baseline was performed using the paired $t$-test. As a secondary analysis, a regression model was constructed to determine the influence, if any, of certain factors (e.g., participants' baseline characteristics) on the change in average number of blocks. The $\chi^{2}$ test or Fisher's exact test $(\mathrm{n}<5$ in any field) was used for comparisons of categorical data. A two-sided $P$ value $<0.05$ was considered statistically significant for the primary outcome.

\section{Results}

From January 2012 through May 2014, 77 participants attended the course and completed 78 pre-course surveys (one participant completed two pre-course surveys by attending two workshops within a 6-month period; only the results of the first survey were retained. This participant had to leave the course early the first time for personal reasons and completed it the second time). The post-course surveys were completed by 46 of 77 (60\%) participants; these 46 pairs of pre- and post-course surveys were included in the analysis. The reported age of the participants $(\mathrm{n}=46)$ on the pre-course survey (median [10th-90th percentiles]) was 50 (37-63) years. There were 38 (83\%) male and $8(17 \%)$ female participants. The time in anesthesia practice was 17 (5-30) years. The time interval from course attendance to follow-up survey completion was 27 (10-34) months.

\section{Primary outcome}

On the post-course survey, participants reported performing 
24 (4-90) blocks per month, on average, compared to 10 (2-24) at baseline $(\mathrm{P}<0.001)$. None of the participants' baseline characteristics (age, sex, follow-up interval in months after course completion, anesthesia experience in years after residency training, UGRA training during residency, and hospital affiliation) were significant cofactors for the change in the average number of blocks.

\section{Secondary outcomes}

After course completion, the number of participants who preferred ultrasound for nerve localization, compared to nerve stimulation alone or ultrasound with nerve stimulation, increased compared to baseline for all of the surveyed block procedures, except for the femoral nerve block (Table 1).

Prior to course participation, the UGRA teaching methods perceived to be most effective were "teaching by colleagues"; other effective teaching methods included "model scanning," "live observation," and "mannequin simulation." At the time of the follow-up, participants' ratings for "mannequin simulation" and "live observation" decreased, whereas ratings for "online materials" increased (Table 2). At follow-up, the leading obstacle to implementing UGRA into clinical practice was time pressure (Table 3). Of the 46 participants, 24 (52\%) reported time pres-

Table 1. Number of Participants Who Preferred Ultrasound as the Sole Nerve Localization Technique (without Concurrent Nerve Stimulation) at Baseline and at Follow-up

\begin{tabular}{lccr}
\hline & $\begin{array}{c}\text { Baseline } \\
(\mathrm{n}=46)\end{array}$ & $\begin{array}{c}\text { Follow-up } \\
(\mathrm{n}=46)\end{array}$ & P value \\
\hline Interscalene & 23 & 31 & 0.005 \\
Supraclavicular & 21 & 34 & $<0.001$ \\
Infraclavicular & 15 & 34 & $<0.001$ \\
Axillary & 19 & 36 & $<0.001$ \\
Femoral & 28 & 33 & 0.166 \\
Sciatic (proximal) & 10 & 30 & $<0.001$ \\
Popliteal-sciatic & 23 & 35 & $<0.001$ \\
Transversus abdominis plane & 16 & 39 & $<0.001$ \\
\hline
\end{tabular}

Values are reported as number of subjects (n), as indicated. P values are based on McNemar's test of correlated proportions. sure as "always" or "almost always" an obstacle, whereas 32/46 (70\%) rated both ultrasound availability and personal preference as "rarely" or "never" an obstacle. At follow-up, 1/46 (2\%) reported with encountered LAST compared to 8/42 (19\%) at baseline $(\mathrm{P}=0.012)$, and $10 / 46(22 \%)$ reported inadvertent vascular puncture without LAST compared to $15 / 42$ (36\%) at baseline ( $\mathrm{P}$ $=0.164)$. Four participants left these questions unanswered on the pre-course survey.

\section{Discussion}

This study demonstrates that a small-group, simulationbased UGRA course with a flexible curriculum addressing specific training needs of practicing anesthesiologists increased self-reported UGRA procedural volume in clinical practice. This change in the number of blocks performed was not influenced by an anesthesiologist's age, years of practical experience, or exposure to UGRA training during residency. This is the first reported study to offer evidence supporting the "practice pathway" recommendations from the American Society of Regional Anesthesia and Pain Medicine (ASRA) and the European Society of Regional Anaesthesia (ESRA) [6] for UGRA training.

A defining feature of the UGRA workshop described here

Table 2. Participants' Rating of Teaching Methods for Learning Ultrasound-guided Regional Anesthesia Based on Perceived Effectiveness at Baseline and at Follow-up

\begin{tabular}{lccc}
\hline & $\begin{array}{c}\text { Baseline } \\
(\mathrm{n}=46)\end{array}$ & $\begin{array}{c}\text { Follow-up } \\
(\mathrm{n}=46)\end{array}$ & P value \\
\hline Lectures & $3(2-4)$ & $4(3-5)$ & 0.074 \\
Model scanning & $4(3-5)$ & $5(4-5)$ & 0.068 \\
Phantom practice & $3(2-5)$ & $4(2-5)$ & 0.110 \\
Live observation & $4(3-5)$ & $4(3-5)$ & $0.042^{*}$ \\
Online materials & $3(3-4)$ & $4(3-5)$ & $0.015^{\dagger}$ \\
Textbooks & $3(2-4)$ & $3(2-4)$ & 0.277 \\
Teaching by colleagues & $5(3-5)$ & $4(4-5)$ & 0.710 \\
Mannequin simulation & $4(3-5)$ & $4(2-5)$ & $0.005^{*}$ \\
\hline
\end{tabular}

Values are reported as median (10th-90th percentiles). *Negative change from baseline to follow-up, ${ }^{\dagger}$ Positive change from baseline to follow-up. Anchored Ordinal Scale 1-5: $1=$ Not Effective. $5=$ Most Effective.

Table 3. Participant Ratings of Factors Identified as Continuing Obstacles to Implementing an Ultrasound-guided Regional Anesthesia Program into Clinical Practice at Follow-up

\begin{tabular}{llcrrr}
\hline \multicolumn{1}{c}{$\mathrm{n}=46$} & Always & Often & Sometimes & Rarely & Never \\
\hline Inadequate skills & $6(13 \%)$ & $6(13 \%)$ & $14(30 \%)$ & $12(26 \%)$ & $8(17 \%)$ \\
Ultrasound availability & $2(4 \%)$ & $3(7 \%)$ & $9(20 \%)$ & $13(28 \%)$ & $19(41 \%)$ \\
Time pressure & $6(13 \%)$ & $18(39 \%)$ & $10(22 \%)$ & $9(20 \%)$ & $3(7 \%)$ \\
No surgeon support & $6(13 \%)$ & $7(15 \%)$ & $21(46 \%)$ & $8(17 \%)$ & $4(9 \%)$ \\
Personal preference & $2(4 \%)$ & $5(11 \%)$ & $7(15 \%)$ & $13(28 \%)$ & $19(41 \%)$ \\
\hline
\end{tabular}

Values are reported as number (\%). 
was the adaptable course content. Compared to the standardized curriculum used in a prior prospective volunteer study, which evaluated a UGRA workshop using simulation to teach perineural catheter placement [12], the format of this non CME, nonresearch educational course was designed to accommodate individual learning objectives and to provide a high expert facultyto-participant ratio. Because each anesthesiologist is unique in his or her training, experience, knowledge, skills, and challenges, this course allowed some flexibility in how time was spent in each session. For example, during the scanning workshops, participants could select block sites and skills that they considered most clinically relevant to their practices, allowing experts to provide individualized instruction and feedback. Similarly, time flexibility encouraged participants to engage in conversations about a range of topics, from anatomy and clinical tips for block success to strategies for implementing clinical pathways and seeking administrative support for new resources and staff. By focusing on challenges that they encountered in their clinical practice, we suggest that participants may have returned to their clinical setting empowered with new knowledge and strategies that facilitated the increase in the number of UGRA procedures at follow up. Another distinguishing feature of this study was the diversity of course participants. Because sponsors extended invitations and selected attendees, practicing anesthesiologists with various levels of experience and expertise in UGRA participated. This differs from the prospective enrollment of volunteer anesthesiologists with minimal-to-no UGRA experience recruited in a previous study [12]. The sample involved in this study may more closely resemble the population of learners who attend current CME or industry-sponsored UGRA courses; thus, the findings of this study may be more generalizable within and outside anesthesiology.

The change in clinical practice, increasing the number of blocks performed per month, was not affected by the time interval between course completion and follow-up. Although our study was not designed to determine the time it takes to effectuate a change in clinical practice or the quality of procedures performed, it does suggest that some participants may acquire new skills and implement them quickly after participating in a 1 day course. Despite the use of simulation to enhance the realism of the training environment [11], any workshop can only offer a limited amount of training in 1 day. However, participants who establish a basic foundation of UGRA-related knowledge and skills may be enabled to perform blocks after course completion. The ASRA-ESRA practice pathway for post-graduate anesthesiologists recommends educational events that offer didactic and hands-on experience for at least $8 \mathrm{~h}$, practice of needle insertion techniques using simulator and phantoms, and spending time with experienced individuals to observe UGRA [12]. While Sites and colleagues [12] noted the "incomplete science surround- ing teaching, learning, and optimal skill acquisition" in UGRA training, this study provides evidence suggesting the beneficial effects of simulation-based practice pathway training in one aspect of clinical practice change in UGRA: increasing the volume of block procedures offered to patients.

After course participation, practicing anesthesiologists preferred to use ultrasound for all of the peripheral nerve block procedures. This represents a change in practice, with the exception of the femoral nerve block, which had the highest rate of ultrasound preference for any block at baseline (28 of 46 participants). The use of ultrasound for peripheral nerve blocks with or without catheter insertion is supported by previous studies demonstrating efficiency, efficacy, and safety advantages [17-21]. The largest changes in nerve localization technique in favor of ultrasound occurred in the proximal sciatic and infraclavicular brachial plexus locations, sites that are often considered "deeper" and potentially more challenging for peripheral nerve blockade using traditional techniques [22,23].

Prior survey based studies investigated the perceived effectiveness of UGRA teaching modalities [13,24]. This study showed that scanning live human models rated highly among participants, both at baseline and follow-up. Live model scanning in the workshop setting cannot be suitably replaced by an online alternative, which likely explains ongoing demand for UGRA courses offered by sponsors or through CME events. However, online education may become more useful to the practicing anesthesiologist as maintenance training. Although mannequin-based simulation in UGRA training has previously been described [8], the results of this study show that ratings decreased at follow-up, compared to baseline. Possible explanations may include limited access to a mannequin or other simulation equipment. Likewise, the ratings for live observation of regional anesthesia procedures decreased at follow-up. Given that participants' reported UGRA procedural volume was higher at the same follow-up time point, we suggest that these ratings may reflect a preference for more hands-on experience rather than additional observation.

Challenges to implementing change in UGRA practice persist despite an overall reported increase in block procedures. For our course participants, the most common reason for not performing UGRA at follow-up was time pressure, which has previously been reported $[11,17,18]$. In addition, lack of support from surgeons is an ongoing institutional challenge reported by course participants. Although anesthesiologists can acquire skills necessary to provide a particular service, introducing a change in clinical practice may involve "buy-in" from other patient providers such as surgeons, nurses, physical and occupational therapists, pharmacists, and even other anesthesiologists [15]. Thus, the process of implementing clinical practice change requires alignment of multiple variables [25]; however, availability 
of ultrasound equipment, once reported as an obstacle to UGRA $[13,24]$, was no longer seen as a common problem in this study.

This study had several limitations. Despite a good survey response rate of $60 \%$, it was vulnerable to sampling bias. Course participants agreed voluntarily to attend the course upon invitation by the sponsor, potentially introducing selection bias in favor of anesthesiologists who were motivated or expected to perform UGRA after returning to their clinical sites. Use of a survey tool did not allow objective assessment of participants' actual UGRA skill level or quality of procedural performance. Fewer occurrences of LAST were identified at follow-up versus baseline. Post-workshop surveys showed participants' greater preference for using ultrasound, and use of ultrasound is associated with a lower occurrence of LAST [19]. Furthermore, we cannot account for other educational sources used or additional workshops attended by the study participants. These factors may have contributed to the participants' natural expansion of their practices. However, very few workshops currently available involve simulation [11], and our previous study showed that simulation was a key element to any UGRA training program involving anesthesiologists in clinical practice [12].
In summary, achieving proficiency in UGRA requires knowledge of anatomy, familiarity with ultrasonography, and technical competency. For practicing anesthesiologists, a 1 day adaptable simulation-based UGRA course that provided extensive faculty interaction and ample hands-on practice can lead to increased UGRA procedural volume in the clinical setting.

\section{Acknowledgments}

Dr. Mariano has received unrestricted educational program funding paid to his institution from Halyard Health (Alpharetta, GA, USA) and B Braun (Bethlehem, PA, USA). These companies had no input into any aspect of the present study design and implementation; data collection, analysis and interpretation; or manuscript preparation.

\section{ORCID}

T. Edward Kim, http://orcid.org/0000-0002-1183-3556 Edward R. Mariano, http://orcid.org/0000-0003-2735-248X

\section{References}

1. Nix CM, Margarido CB, Awad IT, Avila A, Cheung JJ, Dubrowski A, et al. A scoping review of the evidence for teaching ultrasound-guided regional anesthesia. Reg Anesth Pain Med 2013; 38: 471-80.

2. Slater RJ, Castanelli DJ, Barrington MJ. Learning and teaching motor skills in regional anesthesia: a different perspective. Reg Anesth Pain Med 2014; 39: 230-9.

3. Kopacz DJ, Neal JM, Pollock JE. The regional anesthesia "learning curve". What is the minimum number of epidural and spinal blocks to reach consistency? Reg Anesth 1996; 21: 182-90.

4. Sites BD, Gallagher JD, Cravero J, Lundberg J, Blike G. The learning curve associated with a simulated ultrasound-guided interventional task by inexperienced anesthesia residents. Reg Anesth Pain Med 2004; 29: 544-8.

5. Konrad C, Schüpfer G, Wietlisbach M, Gerber H. Learning manual skills in anesthesiology: Is there a recommended number of cases for anesthetic procedures? Anesth Analg 1998; 86: 635-9.

6. Sites BD, Chan VW, Neal JM, Weller R, Grau T, Koscielniak-Nielsen ZJ, et al. The American Society of Regional Anesthesia and Pain Medicine and the European Society of Regional Anaesthesia and Pain Therapy joint committee recommendations for education and training in ultrasound-guided regional anesthesia. Reg Anesth Pain Med 2010; 35(2 Suppl): S74-80.

7. Hocking G, Hebard S, Mitchell CH. A review of the benefits and pitfalls of phantoms in ultrasound-guided regional anesthesia. Reg Anesth Pain Med 2011; 36: 162-70.

8. Niazi AU, Haldipur N, Prasad AG, Chan VW. Ultrasound-guided regional anesthesia performance in the early learning period: effect of simulation training. Reg Anesth Pain Med 2012; 37: 51-4.

9. Rosenberg AD, Popovic J, Albert DB, Altman RA, Marshall MH, Sommer RM, et al. Three partial-task simulators for teaching ultrasoundguided regional anesthesia. Reg Anesth Pain Med 2012; 37: 106-10.

10. Tsui BC, Dillane D, Walji AH. Cadaveric ultrasound imaging for training in ultrasound-guided peripheral nerve blocks: upper extremity. Can J Anaesth 2007; 54: 392-6.

11. Udani AD, Kim TE, Howard SK, Mariano ER. Simulation in teaching regional anesthesia: current perspectives. Local Reg Anesth 2015; 8: $33-43$.

12. Mariano ER, Harrison TK, Kim TE, Kan J, Shum C, Gaba DM, et al. Evaluation of a standardized program for training practicing anesthesiologists in ultrasound-guided regional anesthesia skills.J Ultrasound Med 2015; 34: 1883-93.

13. Ramlogan R, Manickam B, Chan VW, Liang L, Adhikary SD, Liguori GA, et al. Challenges and training tools associated with the practice of ultrasound-guided regional anesthesia: a survey of the American society of regional anesthesia and pain medicine. Reg Anesth Pain Med 2010; 35: 224-6. 
14. Chu LF, Fuller AJ, Mariano ER. A visual guide to regional anesthesia (point of care essentials). Philadelphia, Lippincott Williams \& Wilkins. 2012, pp 1-30.

15. Mariano ER. Making it work: setting up a regional anesthesia program that provides value. Anesthesiol Clin 2008; 26: 681-92.

16. Kim TW, Mariano ER. Updated guide to billing for regional anesthesia (United States). Int Anesthesiol Clin 2011; 49: 84-93.

17. Mariano ER, Loland VJ, Bellars RH, Sandhu NS, Bishop ML, Abrams RA, et al. Ultrasound guidance versus electrical stimulation for infraclavicular brachial plexus perineural catheter insertion. J Ultrasound Med 2009; 28: 1211-8.

18. Mariano ER, Loland VJ, Sandhu NS, Bellars RH, Bishop ML, Afra R, et al. Ultrasound guidance versus electrical stimulation for femoral perineural catheter insertion. J Ultrasound Med 2009; 28: 1453-60.

19. Barrington MJ, Kluger R. Ultrasound guidance reduces the risk of local anesthetic systemic toxicity following peripheral nerve blockade. Reg Anesth Pain Med 2013; 38: 289-99.

20. Marhofer P, Schrögendorfer K, Koinig H, Kapral S, Weinstabl C, Mayer N. Ultrasonographic guidance improves sensory block and onset time of three-in-one blocks. Anesth Analg 1997; 85: 854-7.

21. Dhir S, Ganapathy S. Comparative evaluation of ultrasound-guided continuous infraclavicular brachial plexus block with stimulating catheter and traditional technique: a prospective-randomized trial. Acta Anaesthesiol Scand 2008; 52: 1158-66.

22. Enneking FK, Chan V, Greger J, Hadzić A, Lang SA, Horlocker TT. Lower-extremity peripheral nerve blockade: essentials of our current understanding. Reg Anesth Pain Med 2005; 30: 4-35.

23. Neal JM, Gerancher JC, Hebl JR, Ilfeld BM, McCartney CJ, Franco CD, et al. Upper extremity regional anesthesia: essentials of our current understanding, 2008. Reg Anesth Pain Med 2009; 34: 134-70.

24. O'Sullivan O, Shorten GD, Aboulafia A. Determinants of learning ultrasound-guided axillary brachial plexus blockade. Clin Teach 2011; 8: 236-40.

25. Rangachari P, Rissing P, Rethemeyer K. Awareness of evidence-based practices alone does not translate to implementation: insights from implementation research. Qual Manag Health Care 2013; 22: 117-25. 


\section{Appendix}

\section{Follow-up Survey Instrument}

Name Date of workshop

Phone Number (

Email Address

\section{After attending the workshop (current practice):}

1. For each PNB site, please indicate average number of procedures, preferred nerve localization technique, and comfort level in placing a perineural catheter.

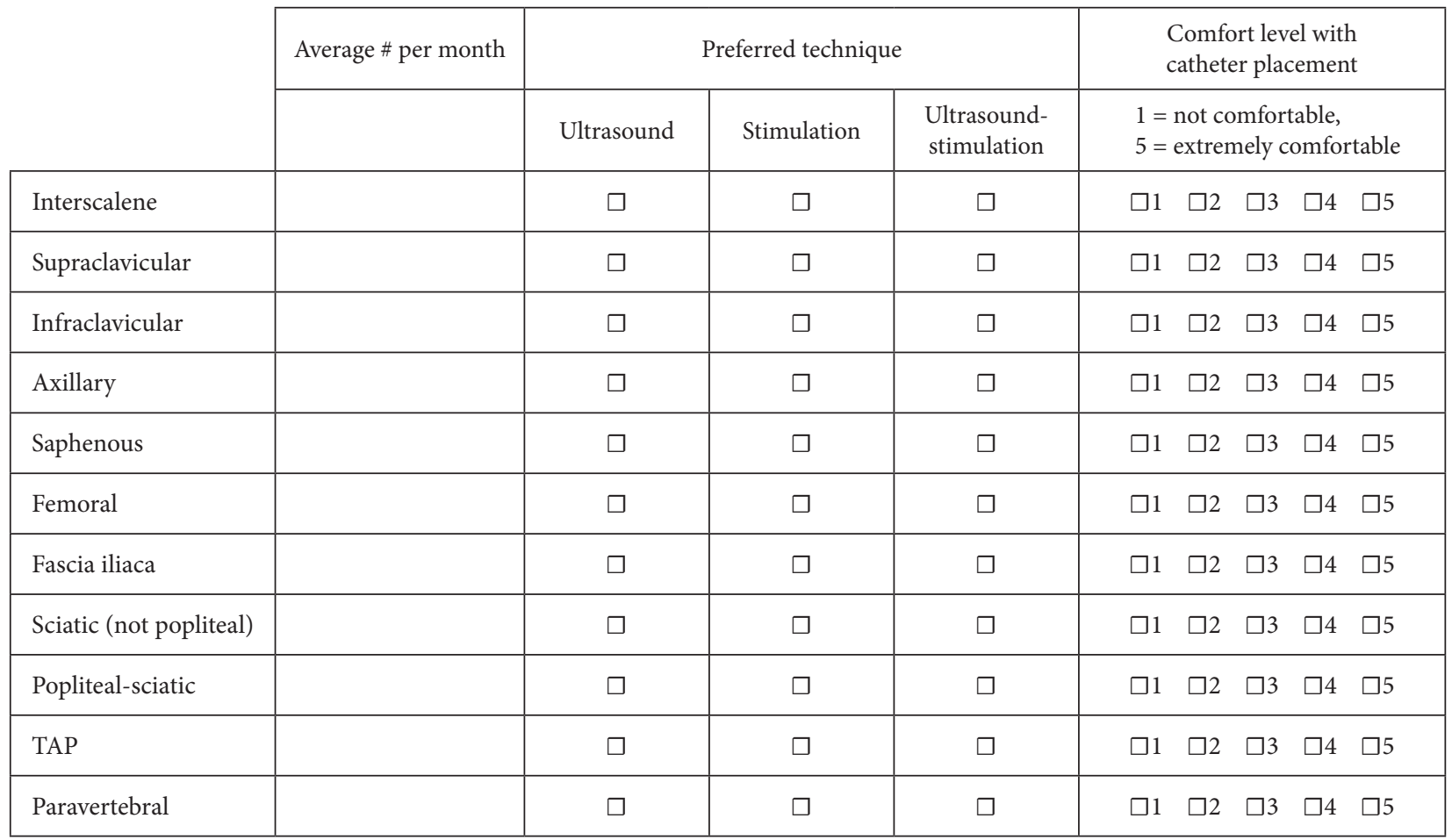

2. Since the workshop, what was your:

Average block success rate (\%):

Number of inadvertent vascular punctures without injection (\#):

Number of intravascular injections resulting in seizures or cardiac arrest (\#): 
3. Of the potential obstacles in implementing an ultrasound-guided perineural catheter program, please rate them using the following 1-5 scale ( 1 = not an obstacle, 5 = biggest obstacle):

$\begin{array}{lcc}\text { Inadequate technical skills } & \square 1 \quad \square 2 \quad \square 3 \quad \square 4 \quad \square 5 \\ \text { Availability of ultrasound equipment } & \square 1 \quad \square 2 \quad \square 3 \quad \square 4 \quad \square 5 \\ \text { Time pressure } & \square 1 \quad \square 2 \quad \square 3 \quad \square 4 \quad \square 5 \\ \text { Support from surgeons } & \square 1 \quad \square 2 \quad \square 3 \quad \square 4 \quad \square 5 \\ \text { Preference for other nerve localization techniques } & \square 1 \quad \square 2 \quad \square 3 \quad \square 4 \quad \square 5\end{array}$

4. Please rate the following teaching methods for US-guided PNB based on your perception of effectiveness since participating in the workshop ( 1 = not effective, $5=$ most effective $)$ :

Scanning on models at a workshop

$\square 1 \quad \square 2 \quad \square 3 \quad \square 4 \quad \square 5$

Phantom practice (e.g., blue phantom or meat)

$\square 1 \quad \square 2 \quad \square 3 \quad \square 4 \quad \square 5$

Observation of real procedures

$\square 1 \quad \square 2 \quad \square 3 \quad \square 4 \quad \square 5$

Online and other electronic tutorials

$\square 1 \quad \square 2 \quad \square 3 \quad \square 4 \quad \square 5$

Textbooks

$\square 1 \quad \square 2 \quad \square 3 \quad \square 4 \quad \square 5$

Teaching by colleagues

$\square 1 \quad \square 2 \quad \square 3 \quad \square 4 \quad \square 5$

Mannequin simulation (immersive learning)

$\square 1 \quad \square 2 \quad \square 3 \quad \square 4 \quad \square 5$

$\square 1 \quad \square 2 \quad \square 3 \quad \square 4 \quad \square 5$ 